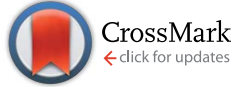

Cite this: J. Mater. Chem. A, 2014, 2 , 13556

Received 6th March 2014 Accepted 16th June 2014

DOI: $10.1039 / \mathrm{c} 4 \mathrm{ta01139b}$

www.rsc.org/MaterialsA

\section{Cellulose nanofibril reinforced composite electrolytes for lithium ion battery applications}

\author{
M. Willgert, ${ }^{a}$ S. Leijonmarck, ${ }^{\text {bc }}$ G. Lindbergh, ${ }^{\text {b }}$ E. Malmström ${ }^{a}$ and M. Johansson*a \\ The present study describes the synthesis and characterization of a series of four composite electrolytes for \\ lithium ion battery applications. The two-phase electrolytes are composed of a soft, ionic conductive \\ poly(ethylene glycol) (PEG) matrix having stiff nanofibrillated cellulose (CNF) paper as reinforcement to \\ provide mechanical integrity. The reinforcing CNF is modified in order to create covalent bonds between \\ the phases which is particularly beneficial when swelling the composite with a liquid electrolyte to \\ enhance the ionic conductivity. After swelling the composite polymer electrolyte, forming a gelled \\ structure, values of ionic conductivity at $5 \times 10^{-5} \mathrm{~S} \mathrm{~cm}^{-1}$ and an elastic modulus around $400 \mathrm{MPa}$ at \\ $25^{\circ} \mathrm{C}$ are obtained.
}

\section{Introduction}

The rapid development of portable devices and electrical vehicles running on electrical energy is calling for new smart ways to store the energy required. ${ }^{1}$ It is a well-known challenge that a large part of the total weight of such items is due to the weight of their batteries. Moreover, batteries are also prone to occupy space within items such as smartphones and laptops, which limits the possibility to make devices possessing small geometries while retaining a suitable performance with respect to energy density, i.e. performance over time. The battery type mainly considered today and for near future applications is the lithium-ion battery due to its high energy density. ${ }^{2}$ Such a battery essentially consists of two electrodes (one anode and one cathode) parted by an electronically insulating separator through which ions can be transported.

One issue that has been addressed regarding lithium batteries over the years concerns their safety; a sudden short circuit might not only make the battery cell useless, but also be detrimental to people and to the environment. ${ }^{3,4}$ To circumvent this it is important to have a robust and efficient separator between the electrodes. One solution to the above-mentioned challenges with lithium batteries is to instead develop what is denoted structural batteries, ${ }^{5}$ where the separator is a polymer not only acting as an ion conducting separator, but also providing other features such as mechanical strength. Using a

${ }^{a}$ KTH Royal Institute of Technology, School of Chemical Science and Engineering, Department of Fibre and Polymer Technology, Teknikringen 56-58, SE-100 44 Stockholm, Sweden. E-mail: matskg@kth.se; Fax: +46 (0)8 79082 83; Tel: +46 (0)8 7909287

${ }^{b}$ KTH Royal Institute of Technology, School of Chemical Science and Engineering, Department of Chemical Engineering and Technology, Applied Electrochemistry, Teknikringen 56-58, SE-100 44 Stockholm, Sweden

${ }^{c}$ Swerea SICOMP AB, Box 104, 43122 Mölndal, Sweden material with high mechanical integrity that also works as a storage medium for electrical energy may solve many issues with respect to weight and space as described above, since the battery can replace a load bearing part in a construction. The use of polymers as the electrolyte media has been investigated $^{5-11}$ since the seventies when D. E. Fenton and P. V. Wright showed that certain polymers can dissolve alkali metals and interact with their corresponding cations. ${ }^{12,13}$ Within the concept of structural- or multi-functional batteries, which have been investigated by many, not least by Snyder et al., the battery can be designed not solely for energy storage, but also to be part of the load bearing construction. ${ }^{14-16}$ This would not only contribute to reduce the total weight and space, which is very much desired in a portable device but also to the long term performance of the battery, especially considering the benefits of having an excellent separator with viscoelastic properties.

These viscoelastic systems typically consist of a crosslinked polymer thermoset known to dissolve lithium salts (i.e. PEGbased thermosets) and the performance regarding ionic conductivity and mechanical properties can be varied with crosslink density. However, in these SPEs with a storage modulus of $>100 \mathrm{MPa}$ at room temperature, the ionic conductivity is fairly low, and does not approach acceptable conductivity levels, i.e. above $10^{-5} \mathrm{~S} \mathrm{~cm}^{-1}$. Since there are substantial limitations on how far homogeneous polymer systems can be pushed without sacrificing performance and ability to satisfy both these criteria, studies have described heterogeneous systems, where one phase provides mechanical performance and the other phase promotes the ionic conductivity, hence making a material with enhanced performance in both aspects. Two-phase systems investigated for electrolyte purposes have included systems reinforced by fibres, inorganic particles, different types of inter-penetrating networks (IPNs) and semiinter penetrating networks (sIPNs). ${ }^{17-19}$ One type of reinforcing 
material that has gained increased attention is cellulose fibril or cellulose nano fibril (CNF), either in composites structures, ${ }^{20-22}$ or as such, together with a liquid electrolyte. ${ }^{23}$ Studies have also presented entire batteries with flexible characteristics con-

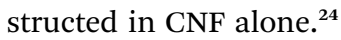

Cellulose is the most abundant natural, renewable polymer and considered to be cheap. At a molecular level it consists of polymer chains having two anhydroglucose rings in each repeating unit (Fig. 1). These polymer chains are gathered into nanofibrils, i.e. CNFs, that are aggregated into macroscopic fibres. In order to extract the fibrils from fibres, pressure is applied in a homogenizing process, which enables the fibrils to be collected. The nanofibrils possess a high aspect ratio, which is beneficial for the reinforcing phase in a composite. ${ }^{25}$ Aggregation is a large problem with untreated CNFs due to their large surface area in relation to volume. In order to decrease the fibre-fibre interaction, and hence the aggregation, additional carboxylate groups can be formed through oxidation of the hydroxymethyl groups on the surface of the fibres through 2,2,6,6-tetramethylpiperidine-1-oxyl radical (TEMPO)-mediated oxidation. ${ }^{26,27}$ This increased charge density on the surface will decrease flocculation and clogging tendency ${ }^{28} \mathrm{CNF}$ possesses a number of interesting features, including superior mechanical strength, where the elastic modulus of a single cellulose nanofibril has been reported to be as high as $140 \mathrm{GPa}^{29,30}$ Their strength and the ability to form paper sheets of high porosity have increased the interest in making strong, permeable electrolytes based on CNFs. When a dispersion of CNFs is dried, a vast number of van der Waals forces and hydrogen bonds are formed between the fibrils. The water removal can be performed in a vacuum funnel, where the CNF slurry is poured onto a filter with small pores. When the residual water is removed through suction filtration, a CNF "cake" remains, which can then be dried, for instance in a vacuum oven. After complete drying, the remaining CNF nanopaper will be dense, brittle and of low porosity. A more porous and ductile paper can be formed if a solvent exchange procedure is employed, where solvents of successively lower polarities are used and filtered through the CNF cake instead of drying it directly.

In the present study the use of functionalized porous CNF nanopapers in structural composite electrolytes is explored as the reinforcing phase in a composite with an ionic conductive polymer matrix. Previous work has addressed the difficulties associated with phase migration when two phases of, for instance, different polarities are to be used in a composite. ${ }^{22,31}$ This also becomes critical if one of the phases changes geometry after manufacturing. In the scope of polymer or gel electrolytes, high ionic conductivity is mostly achieved through

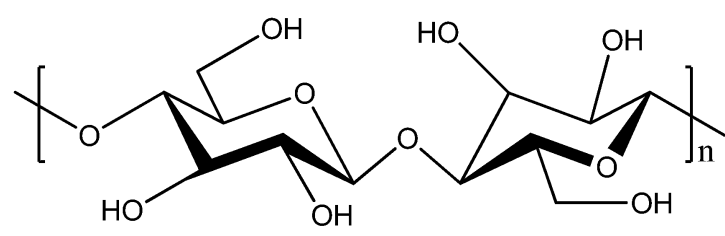

Fig. 1 The repeating unit of cellulose. swelling of the polymer using a liquid electrolyte. Consequently, it is crucial to have a composite that does not deteriorate during the swelling process. The present study addresses this issue via a post-functionalization of the fibril surfaces to enhance the interfacial strength in the final composite electrolyte. The fibrils are modified through an acid chloride reaction to create acrylate and alkyl groups on the surface. The acrylate can react with a PEG-methacrylate to create covalent bonds between the two, and the alkyl will work as a "dummy", keeping the number of unmodified $\mathrm{OH}$-groups constant.

\section{Experimental}

\subsection{Materials}

All reagents and solvents were used as received with no further purification, except for the lithium salt which was dried under vacuum at $80^{\circ} \mathrm{C}$ for two days before entering the glove box.

2.1.1 Materials for preparation of CNF dispersions and CNF nanopapers. A TEMPO oxidation was carried out on CNF fibrils according to the basic procedure described by Saito et $a .^{27}$ First, the never-dried softwood dissolving pulp (40\% Norwegian Spruce, 60\% Scots Pine from Domsjö Fabriker AB, Örnsköldsvik, Sweden) was purified with $0.3 \% \mathrm{NaClO}_{2}$ in an acetate buffer ( $40 \mathrm{~mL}$ of acetate buffer per gram of dry pulp) at $\mathrm{pH} 4.6$ and $60{ }^{\circ} \mathrm{C}$, and stirred for $1 \mathrm{~h}$, followed by a washing step where the pulp was washed with deionized water through filtration. Thereafter, the pulp was re-dispersed in a phosphate buffer ( $90 \mathrm{~mL}$ of phosphate buffer per gram of dry pulp) at $\mathrm{pH}$ 6.8 and $60{ }^{\circ} \mathrm{C} . \mathrm{NaClO}_{2}(10 \mathrm{mmol}, 80 \%)$, TEMPO (0.1 mmol) and $\mathrm{NaClO}(1.0 \mathrm{mmol})$ were calculated per gram of fibre and added to the flask and then the dispersion was stirred for $140 \mathrm{~min}$ at room temperature. At this stage, the pulp was washed with deionized water through filtration. Finally, the charge density of the pulp was determined to be $600 \mu \mathrm{eq} . \mathrm{g}^{-1}$ by conductometric measurement as described by Katz et $a .^{32}$ The pulp was then washed to convert the carboxyl groups to their sodium form before nanopaper preparation according to a procedure previously described by Wågberg et al. ${ }^{33}$ After this pre-treatment, the fibres were homogenized using a high-pressure fluidizer (Microfluidizer M-110EH, Microfluidics Corp.) equipped with two chambers of different sizes connected in series (400 and 200 $\mu \mathrm{m})$. The fibre suspension was passed three times in order to form a gel. This gel was then passed another three times through two pairs of chambers with dimensions 200 and 100 $\mu \mathrm{m}$ respectively. Full homogenization was achieved at a fibre concentration of $1 \mathrm{wt} \%$ and an operating pressure of 1600 bar.

2.1.2 Materials for modification of CNF nanopapers. Acryloyl chloride $\geq 97 \%$ (I), propionyl chloride 98\% (II), 4(dimethylamino)pyridine (DMAP) $\geq 99 \%$, dichloromethane $(\mathrm{DCM}) \geq 99.9 \%$ and silicon dioxide $\left(\mathrm{SiO}_{2}\right)$ 99.5\% of $5-15 \mathrm{~nm}$ particle size were purchased from Sigma-Aldrich. I and II are shown in Fig. 2. Ethanol (EtOH) 99\% and 96\% respectively, acetone $\geq 99.5 \%$ and $n$-pentane $99 \%$ were purchased from VWR International. Triethylamine (TEA) $\geq 99 \%$ and tetrahydrofuran (THF) 99.8\% were purchased from Merck Millipore (Germany).

2.1.3 Matrix material for the composite electrolytes. The PEG-methacrylate oligomers and the lithium salt used are 
<smiles>C=CC(=O)Cl</smiles><smiles>CCC(=O)Cl</smiles>

Fig. 2 The acid chlorides used in modification of the CNF nanopaper: acryloyl chloride (I) and propionyl chloride (II).

depicted in Fig. 3. Tetraethylene glycol dimethacrylate (SR209) (a) $M=330 \mathrm{~g} \mathrm{~mol}^{-1}$ and methoxy polyethylene glycol (350) monomethacrylate (SR550) (b) $M=494 \mathrm{~g} \mathrm{~mol}^{-1}$ were kindly supplied by Sartomer Company, Europe. Lithium hexafluorophosphate $\left(\mathrm{LiPF}_{6}\right)(97 \%)$ was purchased from Chemtronica $\mathrm{AB}$ (Sweden).

The photoinitiator used was 2,2-dimethoxy-2-phenylacetophenone (Irgacure 651) provided by Ciba Specialty Chemicals (Switzerland). For swelling of the composite samples, a $1 \mathrm{M}$ solution of $\mathrm{LiPF}_{6}$ (as mentioned above) in ethyl carbonate (EC) and di-ethyl carbonate (DEC) $(1: 1$ by volume) was prepared.

\subsection{Techniques \& procedures}

2.2.1 Preparation of CNF nanopapers. TEMPO-oxidized CNF (7.5 g of a $1 \mathrm{wt} \%$ water suspension) was placed in a $500 \mathrm{~mL}$ plastic beaker. $75 \mathrm{mg} \mathrm{SiO}_{2}$ was added to the suspension, followed by $90 \mathrm{~g}$ of distilled $\mathrm{H}_{2} \mathrm{O}$. The dilute suspension was stirred at $9000 \mathrm{rpm}$ for 20 minutes using an Ultra Turrax mixer (IKA, D125 Basic). After stirring, the slurry was poured onto a Buchner funnel, having a diameter of $90 \mathrm{~mm}$ equipped with a filter paper with a pore size of $22 \mu \mathrm{m}$, and the water was filtered off overnight, leaving a damp CNF cake in the vacuum funnel. The cake was then subjected to a solvent exchange procedure, where different solvents were subsequently poured over and passed through it as following: EtOH (50 mL, 96\%), EtOH (50 mL, 99\%), dry acetone $(50 \mathrm{~mL})$ and finally pentane $(50 \mathrm{~mL})$. The CNF cake was not allowed to dry completely between successive additions of the different solvents, except for in the first step when the water was passed through prior the addition of EtOH. Once the pentane had passed the filter, the obtained CNF nanopaper was peeled off from the filter and dried under vacuum at $110{ }^{\circ} \mathrm{C}$ overnight in a P Selecta Buch \& Holm Vacuo-temp vacuum oven.

2.2.2 Acrylate and propionate modification of CNF nanopapers. Pieces $\left(1 \mathrm{~cm}^{2}\right)$ of $\mathrm{CNF}$ were cut from the same $\mathrm{CNF}$<smiles>C=C(C)C(=O)OCCOC(C)(C)COC(=O)C(=C)C</smiles>

Fig. 3 The PEG-methacrylate oligomers: tetraethylene glycol dimethacrylate (a), methoxy polyethylene glycol (350) monomethacrylate (b) and $\mathrm{LiPF}_{6}$ used in the study.

\section{Ref}
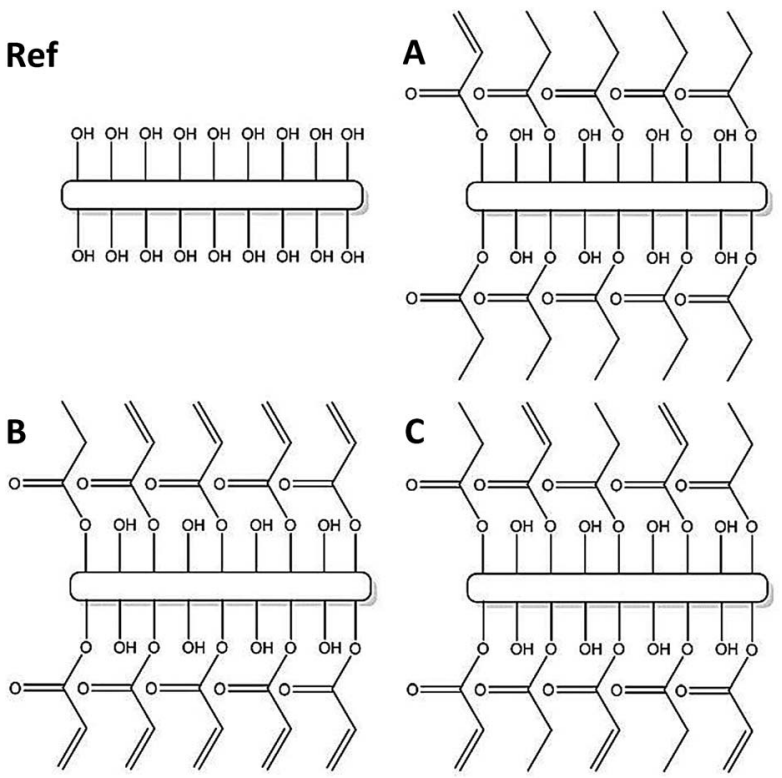

Fig. 4 Schematic description of the four different fibre structures used. It should however be noted that the overall degree of modification of the available $\mathrm{OH}$-groups on the fibre surface in sample Ref, A, $\mathrm{B}$ and $\mathrm{C}$ at present is impossible to quantify.

nanopaper and added into an Erlenmeyer-flask. The samples were then carefully washed with EtOH, acetone, THF and pentane. The samples were ultrasonicated for 2 minutes in each solvent $(10 \mathrm{~mL})$, and afterwards dried in a MMM VACUCELL vacuum oven for 12 hours at $55{ }^{\circ} \mathrm{C}$. Each sample was put in separate vials, and DCM $(10 \mathrm{~mL})$ was added, followed by TEA ( 34 $\mu \mathrm{L}(0.24 \mathrm{mmol}))$ and a catalytic amount of DMAP. Finally, a mixture of acryloyl chloride/propionyl chloride was added dropwise to samples A-C according to the ratios presented in Table 1 (Fig. 4). No acid chlorides were added to the vials containing the reference samples (Ref1 and 2), which otherwise were treated identical to the other samples. The reaction mixture was left on a shaking table and left to react overnight at room temperature. After the reaction, the solution was poured off and the pieces of CNF were washed thoroughly with EtOH, THF, DCM and pentane, and sonicated in each solvent for 2 minutes. Finally, the samples were dried in a vacuum oven for 12 hours at $55{ }^{\circ} \mathrm{C}$.

Table 1 Details of modification solutions used ${ }^{a}$

\begin{tabular}{llll}
\hline & \multicolumn{2}{l}{ Acid chloride } & \\
\cline { 2 - 4 } Sample name & $\begin{array}{l}\text { Acryloyl chloride } \\
(\mathbf{I})\end{array}$ & $\begin{array}{l}\text { Propionyl chloride } \\
(\mathbf{I I})\end{array}$ & $\begin{array}{l}\text { Stoichiometry } \\
(\mathbf{I}: \text { II })\end{array}$ \\
\hline Ref1 and 2 & - & - & $0: 0$ \\
A1, A2 & $(0.2 \mathrm{mmol})$ & $(2 \mathrm{mmol})$ & $1: 9$ \\
B1, B2 & $(2 \mathrm{mmol})$ & $(0.2 \mathrm{mmol})$ & $9: 1$ \\
C1, C2 & $(1.1 \mathrm{mmol})$ & $(1.1 \mathrm{mmol})$ & $1: 1$
\end{tabular}

${ }^{a}$ All sample vials contained $34 \mu \mathrm{L}(0.24 \mathrm{mmol})$ TEA and a catalytic amount of DMAP. 
2.2.3 Composite electrolyte preparation and photopolymerization. The following general procedure was used for the preparation and curing of the polymer composite electrolytes. The PEG methacrylate oligomers (5 wt\% of a and $95 \mathrm{wt} \%$ of b) were mixed in vials in a glove box under dry conditions $(<1$ ppm $\mathrm{H}_{2} \mathrm{O}$ ) under argon. The dried lithium salt (8 wt\% of the total oligomer weight) and the photoinitiator ( $2 \mathrm{wt} \%$ of the total oligomer weight) were added and dissolved in the mixture. The vials were sealed and placed on a shaking table overnight, allowing the lithium salt and the photoinitiator to dissolve completely.

The modified and unmodified CNF pieces were immersed in a vial containing the monomer mixture, and the vial was connected to a vacuum/argon line. Vacuum was then applied until no bubbles were observed, and the appearance of paper had turned from white to transparent.

At that moment, argon was allowed to enter the vial by increasing the pressure back to normal atmospheric pressure. This cycle was repeated three times to allow complete removal of entrapped air as well as the remaining oxygen. When this was accomplished, the vial containing the monomer mixture as well as the CNF piece was moved inside the glove box. The wet CNF piece was lifted out from the mixture and put between two polyester plastic films (Mylar®). Finally, a microscope lab glass slide was put on top and the sample was irradiated for 10 minutes on one side +3 minutes on the other under UV irradiation at $15 \mathrm{~cm}$ distance from the UV light source, and the resulting, fully cured, still transparent composite electrolyte film was peeled off the Mylar® films. The temperature of the sample did not exceed $42{ }^{\circ} \mathrm{C}$ during cure. The light source used for curing was a Blak Ray B-100AP (100 W, 365 nm) Hg UV lamp, which after the aforementioned irradiation time subjects the sample to a total dose of $4.1 \mathrm{~J} \mathrm{~cm}^{-2}$, as determined using an Uvicure Plus High Energy UV Integrating Radiometer (EIT, USA), measuring UVA at 320-390 $\mathrm{nm}$. After irradiation, the composite electrolyte was swollen in EC : DEC $(1: 1)$ for two hours, after which different characterizations were conducted. Additionally, two neat polymer reference samples without any CNF were manufactured. One of them was characterized as such and the other was swollen as described above before characterization.

2.2.4 Electrochemical impedance spectroscopy (EIS). The electrochemical performances of the composite electrolytes were quantified using a two-electrode test cell, consisting of two stainless steel block electrodes in which between two copper gauge blocks $(6.5 \times 6.5 \times 1 \mathrm{~mm})$ the sample $(10 \times 10 \times 0.15$ $\mathrm{mm}$ ) was mounted. The copper blocks were used in order to make sure that the measurements were actually taking place through the composite electrolyte, and not through the excess polymer matrix on the edges and/or the liquid electrolyte (Fig. 5). The impedance was measured around open circuit potential (OCP) in the frequency range $0.1 \mathrm{~Hz}$ to $300 \mathrm{kHz}$, at 10 points per decade using an amplitude of $10 \mathrm{mV}$. The instrument utilized was a Gamry Series G 750 Potentiostat/Galvanostat/ZRA interface, in an argon-filled glove box $\left(<1\right.$ ppm $\left.\mathrm{H}_{2} \mathrm{O}\right)$ at $25{ }^{\circ} \mathrm{C}$.

2.2.5 Dynamical mechanical analysis (DMA). To quantify the mechanical performance of the composite electrolyte,

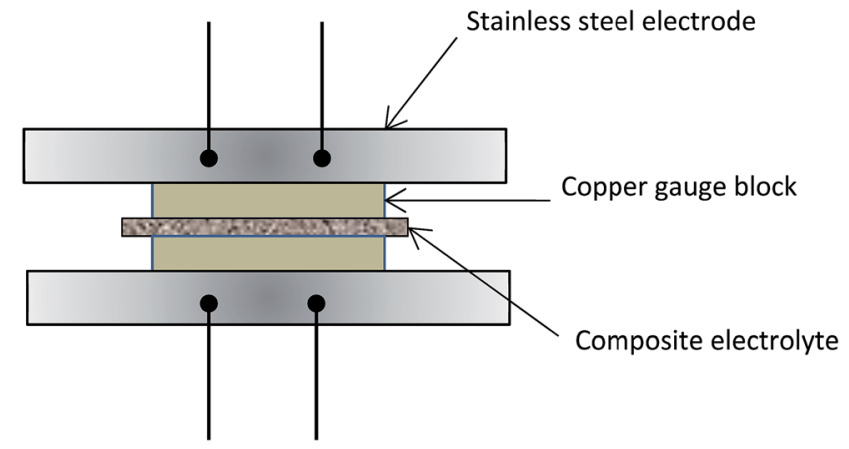

Fig. 5 A schematic figure of the EIS setup.

dynamical mechanical analysis (DMA) tests were performed on a TA instruments DMA, model Q800 in tensile mode. The composite electrolyte sample pieces for DMA measurements possessed a geometry of $5 \times 20 \times 0.15 \mathrm{~mm}$. The specimens were tightened in the clamps of the sample holder, and the temperature was then decreased to and held at the starting temperature $\left(-60{ }^{\circ} \mathrm{C}\right)$ for 5 minutes before the measurements were started. The temperature was then increased by $5{ }^{\circ} \mathrm{C} \mathrm{min}^{-1}$ up to $130{ }^{\circ} \mathrm{C}$ as data were recorded. The oscillation frequency was held at $1 \mathrm{~Hz}$ at a constant amplitude of $10.0 \mu \mathrm{m}$. DMA measurements gave values for storage modulus $\left(E^{\prime}\right)$ at $25{ }^{\circ} \mathrm{C}$, loss modulus $\left(E^{\prime \prime}\right)$, loss factor $(\tan \delta)$ and $T_{\mathrm{g}}$ for the electrolytes presented in Table 2.

2.2.6 Fourier-transform infrared (FT-IR) spectroscopy. The CNF nanopapers were characterized by FT-IR prior to and after modification, to verify the presence of acrylate and propionate groups. Furthermore, the composite electrolyte samples were also characterized after curing to make sure that the composites were fully cured. The whole FT-IR analysis was performed using a Perkin-Elmer Spectrum 2000 FT-IR instrument (Norwalk, CT) equipped with a single reflection (ATR: attenuated total reflection) accessory unit having a diamond ATR crystal (Golden Gate) from Graseby Specac Ltd. (Kent, England). The penetration depth is circa 1-5 $\mu \mathrm{m}$ depending on the wavenumber. Spectrum software was utilized to evaluate the data. All IR measurements were performed in reflection mode, with a resolution of $4 \mathrm{~cm}^{-1}$. Free standing composite electrolyte films were obtained by peeling off from the Mylar ${ }^{\circledR}$ films and mounted onto the instrument, with the bottom side of the sample (exposed to 3 minutes of irradiation) facing the crystal. The disappearance of the vinyl stretch peak at $1637 \mathrm{~cm}^{-1}$ was confirmed after $10+3$ minutes of UV irradiation. Each spectrum was based on 32 scans.

2.2.7 Field-emission scanning electron microscopy (FESEM). The fracture surfaces of the cured composite electrolytes were examined by SEM using a Hitachi S-4800 equipped with a cold field-emission electron source. Images were captured for samples Ref, A and B to examine the coverage of the polymer in the CNF nanopapers. The samples were cut using a scalpel through the composite electrolyte. The fracture surfaces of all samples were coated with Pt/Pd using Agar HR sputter coaters and the sputtered layers were around $5 \mathrm{~nm}$ thick. 
Table 2 Summary of results obtained for the test series ${ }^{a b c}$

\begin{tabular}{lclll}
\hline Sample name & wt\% CNF & $E_{25 \circ \mathrm{C}}^{\prime}[\mathrm{MPa}]$ & $E_{100 \circ \mathrm{C}}^{\prime}[\mathrm{MPa}]$ & $\sigma_{25^{\circ} \mathrm{C}}\left[\mathrm{S} \mathrm{cm}{ }^{-1}\right] \times 10^{-5}$ \\
\hline CNF nanopaper & 100 & 500 & 435 & $\mathrm{n} / \mathrm{a}$ \\
Polymer matrix reference (dry) & 0 & 3 & 3 & $\mathrm{n} / \mathrm{a}$ \\
Polymer matrix reference (swollen) & 0 & 4 & $\mathrm{n} / \mathrm{a}$ & $\mathrm{n} / \mathrm{a}$ \\
Ref1 (unmodified) & 47 & 710 & 330 & 1.2 \\
Ref2 (unmodified) & 47 & 690 & 300 & 1.8 \\
A1 acrylate : propionate $(1: 9)$ & 36 & 440 & 100 & 7.9 \\
A2 acrylate : propionate $(1: 9)$ & 36 & 570 & $\mathrm{n} / \mathrm{a}$ & 6.6 \\
B1 acrylate : propionate $(9: 1)$ & 40 & 470 & $\mathrm{n} / \mathrm{a}$ & 1.4 \\
B2 acrylate : propionate $(9: 1)$ & 40 & n/a & 160 & 1.1 \\
C1 acrylate : propionate $(1: 1)$ & 40 & 520 & 120 & 7.0 \\
C2 acrylate : propionate $(1: 1)$ & 40 & 350 &
\end{tabular}

${ }^{a}$ The density of the CNF nanopaper used in all composites is $618 \mathrm{~kg} \mathrm{~m}^{-3} \cdot{ }^{b}$ The porosity of the CNF nanopaper used in all composites is $58 \%$ (eqn (1)). ${ }^{c}$ The neat polymer matrix samples were too brittle to undergo EIS measurements without breaking.

2.2.8 Swelling tests in EtOH. The stability of the composite electrolytes towards solvent was evaluated by swelling tests in ethanol. The samples were immersed in $\mathrm{EtOH}$ and put on a shaking table for 48 hours. The samples were visually assessed with respect to transparency i.e. an increased opaqueness indicates a weak fibre/matrix interface.

2.2.9 Porosity and density measurements on the CNF nanopaper. The density of the CNF nanopaper used was determined by measuring and dividing the weight by the volume of the sample. The volume was calculated from the thickness of the CNF nanopaper and its area (both measured with a digital slide caliper). The porosity was then calculated using eqn (1) where the density value of the cellulose is chosen to be $1460 \mathrm{~kg} \mathrm{~m}^{-3} \cdot{ }^{34}$

$$
\text { porosity }[\%]=100 \times\left(1-\frac{\rho_{\mathrm{CNF} \text { nanopaper }}}{\rho_{\text {cellulose }}}\right)
$$

2.2.10 Calculation of the amount of CNFs as part of the composite electrolyte. The weight fraction of CNFs in the composites (Table 2) is calculated from the weight of the unmodified CNF nanopaper and the composite manufactured from the same paper.

2.2.11 Cyclic voltammetry. For the cyclic voltammetry (CV) measurements, on sample $\mathrm{C} 1$, the experiments were carried out on a Gamry G 750 potentiostat at a sweep rate of $1 \mathrm{mV} \mathrm{s}^{-1}$, with the software recording data each second. The measurement was performed from the open circuit potential (OCP), sweeping down to $1 \mathrm{mV} v s$., $\mathrm{Li} / \mathrm{Li}^{+}$, then up to $6 \mathrm{~V} v s$. $\mathrm{Li} / \mathrm{Li}^{+}$, and finally down to the original OCP again.

\section{Results and discussion}

The present study includes a series of CNF paper based composite electrolytes with different functionalities on the cellulose fibre surfaces to be used in structural lithium ion battery applications. Neat CNF paper as well as modified were used as reinforcement for the composite electrolytes. The modifications were conducted with different ratios of reactive or inert moieties in order to reveal the influence of both chemical and physical interactions between the reinforcing fibres and the matrix.

Data on the sample compositions are found in Table 1. A summary of the obtained data for the electrolyte composites and their precursors is presented in Table 2.

\subsection{Modification of CNF nanopapers}

Fig. 6 shows the FT-IR spectra of neat CNF paper and after modification (B1) with the acid chlorides used in this study. It can clearly be seen that after modification, a peak at $1740 \mathrm{~cm}^{-1}$ appears corresponding to the carbonyl group of an ester. ${ }^{35}$ This suggests a successful modification however, the complex morphology of the fibrillar structure as well as the porous character of the system do not allow for a quantitative determination of the amount of surface modification. It is not possible to quantify, or even detect, the double bonds on the substrate due to the intrinsic low absorptivity of the acrylate double bond $\left(1640 \mathrm{~cm}^{-1}\right)$ and the overlap with the peak emanating from trace amounts of water bound to cellulose.

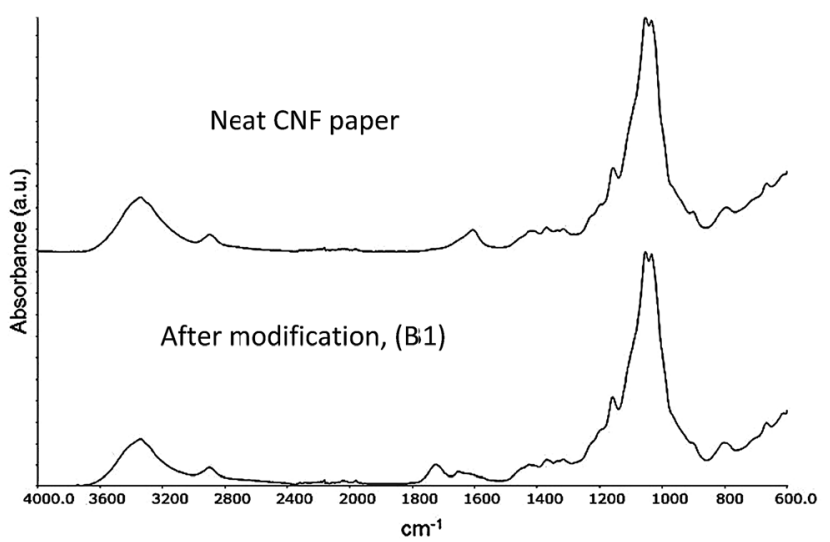

Fig. 6 FT-IR spectra of sample B1 before (top) and after (bottom) modification. 


\subsection{Composite electrolyte formation}

The modified nanopapers were placed in a vial containing the PEG-oligomer solution. The air entrapped in the pores of the nanopaper was removed under vacuum and subsequently replaced by the solution, transforming the nanopaper from white to transparent, Fig. 7.

The transparent nature of the impregnated nanopaper indicates a complete removal of entrapped air. It further implies either that the difference in refractive index between the fibres and the matrix is small and/or that the domain sizes in the different phases are smaller than the wavelength of visible light.

\subsection{Curing performance}

As described in previous studies the UV curing method works well for manufacturing of PEG-methacrylate based composite electrolyte films in the presence of a lithium salt. ${ }^{20,36,37}$ This approach was found to be versatile for the nanopaper reinforced composite electrolytes investigated in this study. Transparent, flexible, smooth, and easy-to-handle free-standing films were obtained after curing, Fig. 8. The success of the curing reaction was confirmed using FT-IR, monitoring the disappearance of the peak corresponding to the vinyl bond stretch in the methacrylate group of the oligomers (at $\sim 1640 \mathrm{~cm}^{-1}$ and $\sim 815$ $\mathrm{cm}^{-1}$ ). The FT-IR spectra, Fig. 8, show the absorption of the uncured oligomer mixture, sample Ref1 after curing, and sample B1 after curing and swelling, verifying full conversion within the detection limit of the FT-IR technique.

\subsection{Properties of the composite electrolyte}

After curing, smooth, non-sticky, transparent composite electrolytes were obtained (Fig. 9).

The readily formed free-standing films were easy to handle, even after post-swelling with a liquid electrolyte, and easily mounted in the characterization instruments used.

3.4.1 Mechanical properties of the composite electrolytes. The results of the mechanical analysis obtained are shown in Fig. 10. When looking at the elastic modulus curve for the unreinforced polymer compared to the reinforced counterparts, it is readily seen that the storage modulus is increased significantly when the matrix is reinforced with nanopaper. The modulus increases nearly 600 times compared to the unreinforced polymer, above its $T_{\mathrm{g}}$.
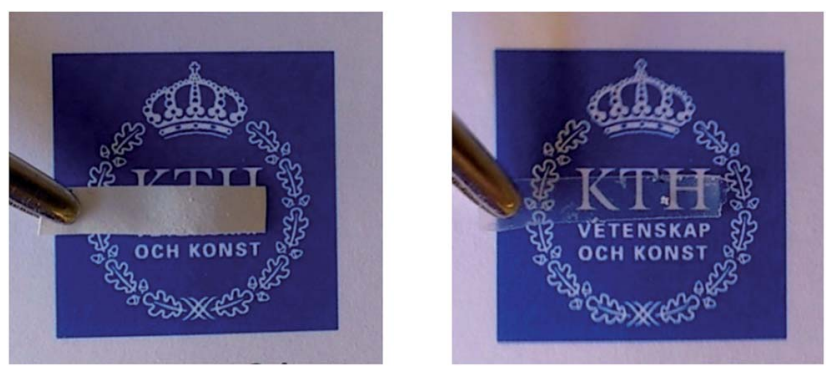

Fig. 7 After removing the entrapped air the paper becomes transparent prior to curing.

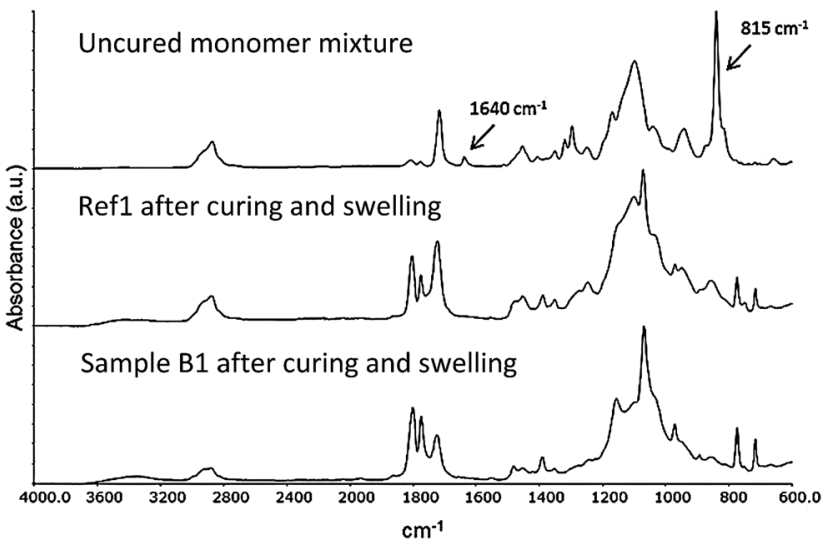

Fig. 8 FT-IR spectra of the uncured monomer mixture (top), sample Ref1 (middle) and sample B1 (bottom) after curing and swelling.

The pure polymer matrix reference exhibits a low $T_{\mathrm{g}}$ around $-20{ }^{\circ} \mathrm{C}$ with a very low modulus in the rubbery state. The pure CNF paper on the other hand exhibits no detectable phase transition and a relatively high modulus throughout the measurement range. The composite materials all exhibit two phase transitions; one low, corresponding to the pure matrix's $T_{\mathrm{g}}$ and the other transition around $50{ }^{\circ} \mathrm{C}$. This higher transition is proposed to originate from an interphase region in the material i.e. the polymer in close proximity to the fibre surfaces. $^{38}$ Comparing the unmodified CNF paper with the modified counterpart it is observed that the unmodified exhibits a higher modulus. This can be explained by stronger interactions between the reinforcing fibres when unmodified.

Sample B1, having a large amount of acrylate modification, exhibited a brittle behavior leading to mechanical failure around $60{ }^{\circ} \mathrm{C}$. A plausible explanation is that a combination of high crosslink density at the fibre surfaces and the post-swelling with EC : DEC induces stresses at the interface with a subsequent loss in ductility.

Furthermore, it can be interpreted from these results that in the sample with the lowest amount of acrylates, relatively poor mechanical performance is obtained compared to the sample with an equal amount of acrylate and propionate groups.

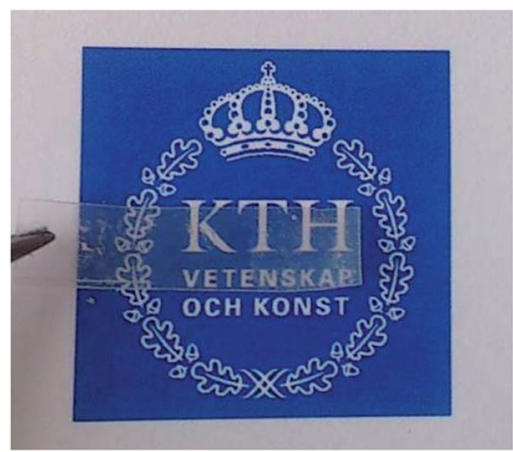

Fig. 9 The cured composite electrolyte product. 


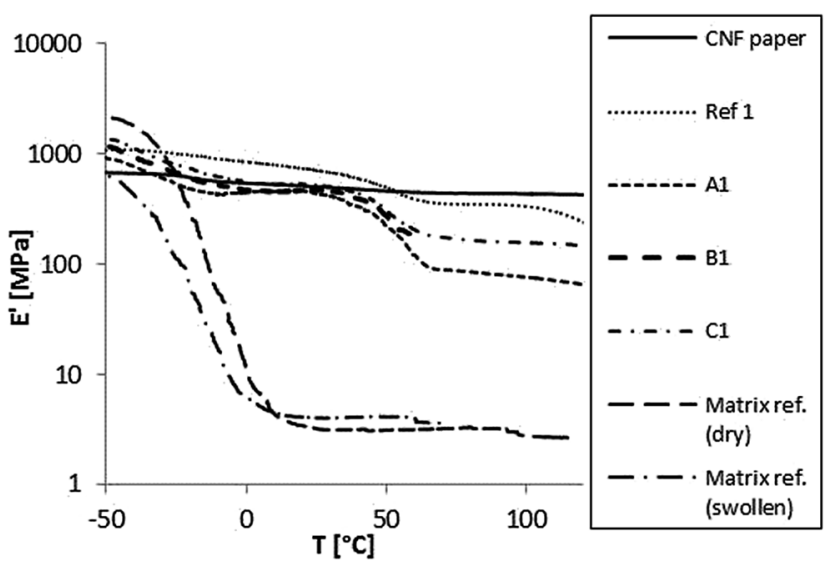

Fig. $10 E^{\prime}$ as a function of temperature for the CNF reinforced composites having varying amounts of acryloyl : propionyl modification, as well as the results for the neat polymer and CNF nanopaper respectively.

All together the results imply that if the interfacial bonds are too few, the polymer is more mobile and undergoes its glass transition with no restraints from the CNF. When the bonds are too many, the composite becomes brittle. With an equimolar modification (samples C1 and C2) the modified composite exhibits an excellent modulus ( $>100 \mathrm{MPa})$, even above $100{ }^{\circ} \mathrm{C}$, in its swollen state.

3.4.2 Ion conducting properties. All samples were evaluated using electrochemical impedance spectroscopy, even though the neat polymer matrix samples and the swollen counterparts were too fragile to give trustworthy measurements. The results presented in Fig. 11 show that all composite electrolyte samples exhibit conductivity values $>10^{-5} \mathrm{~S} \mathrm{~cm}^{-1}$ and all in the same decade. However, it can be noted that the A1 samples having the $1: 9$ composition (acryloyl chloride : propionyl chloride) have a value of almost a decade higher than the reference samples.

The samples based on unmodified CNF (Ref1 and 2) measure lower due to the fact that the polymer has left the composite during the swelling process and ionic pathways are obstructed. When considering the samples with a larger amount of acrylate groups, their ionic transporting ability is reduced. This is most

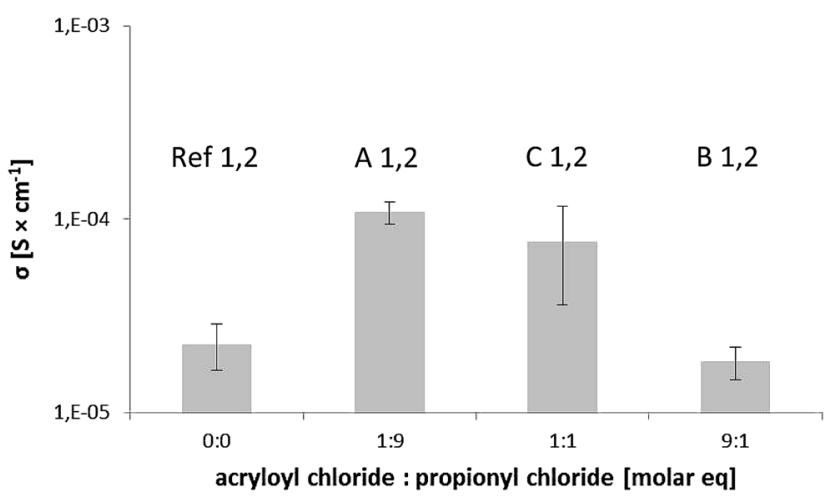

Fig. 11 Ionic conductivities for all samples at $25^{\circ} \mathrm{C}$. likely due to the limited segmental mobility of the PEG segments as a consequence of the covalent bonds between the phases. Furthermore, samples Ref1 and 2 show a more moderate ionic conductivity as well indicating a strong fibre/ matrix interaction in the solid state.

3.4.3 Swelling tests in EtOH. In order to obtain an assessment of to what extent the ion conducting polymer was covalently linked to the CNF, swelling tests were conducted. All samples were immersed in EtOH and left on a shaking table for 48 hours. It was anticipated that since EtOH is an excellent solvent for PEG polymers, a larger amount of PEG-polymer was expected to migrate from the unmodified CNF nanopaper than from the modified. After 48 hours, the sample with unmodified CNF turned opaque again, while the sample with modified CNF's retained a higher transparency (Fig. 12).

This can be interpreted so that a larger amount of polymer is removed from the unmodified CNF paper than from the modified counterpart. This is a strong indication that the polymer is covalently attached to the CNF in the latter case. These results furthermore indicate the importance of a strong interface in the composite material when the material is subjected to swelling stresses.

The possibility of the polymer electrolyte to diffuse out of the system will be important for the long term performance of the composite electrolyte in a battery application. Not only will the load bearing ability be affected but also the electrochemical behavior will deteriorate with increased porosity. Changes in geometry have also imposed in a battery during charging/ uncharging cycles why a strong interface is needed. This study suggests that a strong interface can be obtained when the ion conducting matrix is covalently anchored.

3.4.4 Composite electrolyte morphology. In order to investigate the morphology of the composite electrolytes, SEM imaging was conducted. Fig. 13a-d show representative images of a plain CNF sample, Ref1, A1 and B1. In sample Ref1 the wetting of the CNF is poor when fractured, as seen by segments of naked CNF fibres. On the other hand, when fracturing the composites having the matrix covalently linked to the CNF fibres, it can be observed that essentially no naked fibres are seen. This further emphasizes the importance of a strong

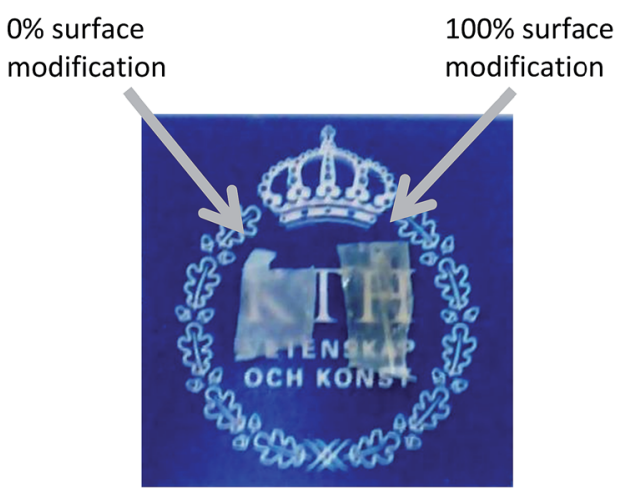

Fig. 12 The composite electrolyte with unmodified CNF nanopaper (left) and modified CNF nanopaper (right) after swelling in EtOH for 48 hours. 

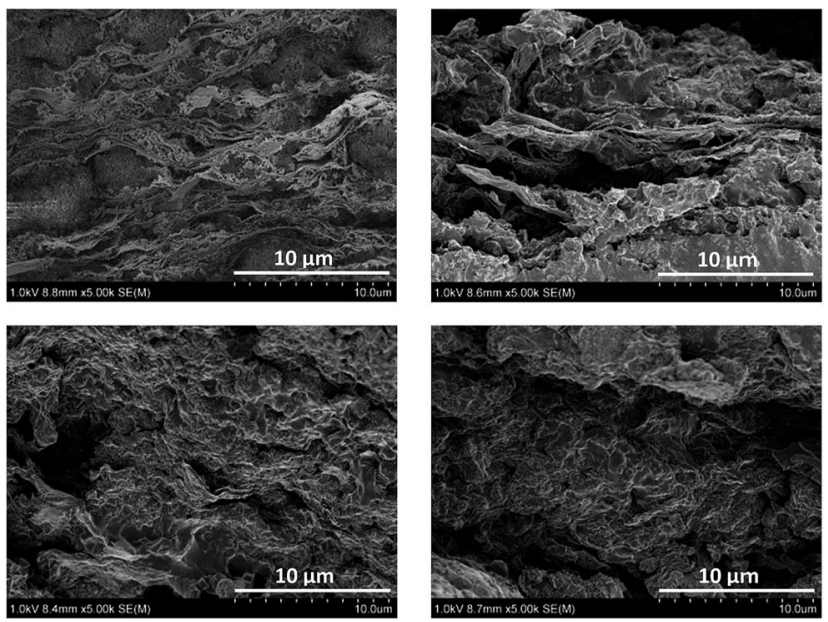

Fig. 13 (a-d) SEM images of sample plain CNF (upper left), Ref1 (upper right), A1 (lower left) and B1 (lower right).

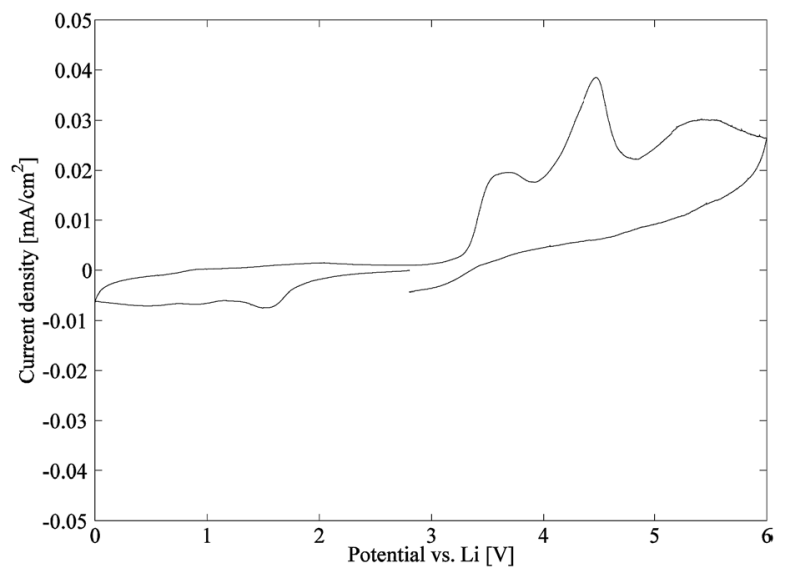

Fig. 14 Cyclic voltammetry plot of sample C1.

interface when the composites are subjected to dimensional changes as will be expected in a working lithium ion battery.

3.4.5 Cyclic voltammetry. To evaluate the electrochemical stability for the swelled composite electrolytes, a CV test was performed between $1 \mathrm{mV}$ and $6 \mathrm{~V}$ vs. Li metal. The resulting currents $\left(<40 \mu \mathrm{A} \mathrm{cm}{ }^{-2}\right)$ illustrate a relatively stable electrochemical behavior (Fig. 14), with no major break-down of the electrolyte materials. As all materials in the composite have been used in Li-ion battery testing before ${ }^{\mathbf{2 4 , 3 7}}$ the results are not surprising.

\section{Conclusions}

In this study, a series of 4 composite electrolytes has been successfully manufactured. The composite electrolytes are constituted of a soft, ion conducting polymer matrix having a stiff CNF nanopaper as reinforcement. The reference samples contain unmodified CNFs, while the other samples in the series are surface modified by a straightforward reaction to create covalent bonds between the CNF and the polymer matrix to different extents. After swelling the composite electrolytes with liquid electrolytes, the sample with the lowest amount of acrylate modification is the best ionic conductor. However, when both the mechanical properties and ion conducting performance are considered, the samples modified by an equimolar solution of propionyl chloride and acryloyl chloride perform best, having an elastic modulus $>100 \mathrm{MPa}$ above $100{ }^{\circ} \mathrm{C}$ and an ion conductivity of around $5 \times 10^{-5} \mathrm{~S} \mathrm{~cm}^{-1}$ at $25{ }^{\circ} \mathrm{C}$. In conclusion, these promising results by far meet the criteria set up by Snyder et al. for structural batteries $\left(E^{\prime}>150 \mathrm{MPa}, \sigma>1 \times\right.$ $\left.10^{-5} \mathrm{~S} \mathrm{~cm}^{-1}\right),{ }^{14}$ and further studies regarding these composites are currently undertaken. Finally, the resulting currents from the cyclic voltammetry measurements illustrate a relatively stable electrochemical behaviour of the composite electrolyte.

\section{Acknowledgements}

The Swedish Energy Agency is greatly acknowledged for financial support, grant \#37712-1 "Structural composite batteries for energy efficient vehicles". The monomers used were supplied by Sartomer Company which is thankfully acknowledged. Linn Carlsson and Nicholas Cervin are acknowledged for providing CNFs and Christian Porsch for valuable discussions and input on this article.

\section{Notes and references}

1 E. D. Wetzel, Reducing weight: Multifunctional composites integrates power, communications, and structure, AMPTIAC Q., 2004, 8(4), 91-95.

$2 \mathrm{~J}$. M. Tarascon and M. Armand, Issues and challenges facing rechargeable lithium batteries, Nature, 2001, 414(6861), 359367.

3 R. Spotnitz and J. Franklin, Abuse behavior of high-power, lithium-ion cells, J. Power Sources, 2003, 113(1), 81-100.

4 B. Yebka, J. A. Holung and T.-L. Wong, Battery safety features, Lenovo (Singapore) Pte. Ltd., Singapore, 2010, U.S. Pat. Appl. Publ. US., p. 11.

5 P. Liu, E. Sherman and A. Jacobsen, Design and fabrication of multifunctional structural batteries, J. Power Sources, 2009, 189(1), 646-650.

6 M. B. Armand, M. J. Duclot and P. Rigaud, Polymer solid electrolytes: stability domain, Solid State Ionics, 1981, 3-4, 429-430.

7 M. Armand, Polymer solid electrolytes - an overview, Solid State Ionics, 1983, 9-10(pt 2), 745-754.

$8 \mathrm{C}$. Berthier, et al., Microscopic investigation of ionic conductivity in alkali metal salt-poly(ethylene oxide) adducts, Solid State Ionics, 1983, 11(1), 91-95.

9 D. F. Shriver, et al., Structure and ion transport in polymersalt complexes, Solid State Ionics, 1981, 5, 83-88.

$10 \mathrm{G}$. Bao, et al., Ionic conductivity of polyelectrolyte derivatives of poly(vinyl alcohol) - lithium ion complex films, Polym. Bull., 1987, 18(2), 143-148.

11 Y. Choi, et al., Ultraviolet radiation curing of acrylates for lithium polymer electrolytes, J. Appl. Electrochem., 1997, 27(9), 1118-1121. 
12 D. E. Fenton, J. M. Parker and P. V. Wright, Complexes of alkali metal ions with poly(ethylene oxide), Polymer, 1973, 14(11), 589.

13 P. V. Wright, Electrical conductivity in ionic complexes of poly(ethylene oxide), Br. Polym. J., 1975, 7(5), 319-327.

14 J. F. Snyder, R. H. Carter and E. D. Wetzel, Electrochemical and Mechanical Behavior in Mechanically Robust Solid Polymer Electrolytes for Use in Multifunctional Structural Batteries, Chem. Mater., 2007, 19(15), 3793-3801.

15 J. F. Snyder, E. D. Wetzel and C. M. Watson, Improving multifunctional behavior in structural electrolytes through copolymerization of structure- and conductivity-promoting monomers, Polymer, 2009, 50(20), 4906-4916.

16 J. P. Thomas and M. A. Qidwai, The design and application of multifunctional structure-battery materials systems, $J$. Occup. Med., 2005, 57(3), 18-24.

$17 \mathrm{X}$. Hou and K. S. Siow, Ionic conductivity and electrochemical characterization of novel interpenetrating polymer network electrolytes, Solid State Ionics, 2002, 147(3-4), 391-395.

18 D. He, et al., Enhanced Ionic Conductivity of Semi-IPN Solid Polymer Electrolytes Based on Star-Shaped Oligo(ethyleneoxy)cyclotriphosphazenes, Macromolecules, 2012, 45(19), 7931-7938.

19 P. Meneghetti, S. Qutubuddin and A. Webber, Synthesis of polymer gel electrolyte with high molecular weight polymethyl methacrylate-clay nanocomposite, Electrochim. Acta, 2004, 49(27), 4923-4931.

20 J. R. Nair, et al., UV-cured polymer electrolyte membranes for Li-cells: Improved mechanical properties by a novel cellulose reinforcement, Electrochem. Commun., 2009, 11(9), 17961798.

21 A. Chiappone, et al., Microfibrillated cellulose as reinforcement for Li-ion battery polymer electrolytes with excellent mechanical stability, J. Power Sources, 2011, 196(23), 10280-10288.

22 J. R. Nair, et al., Novel cellulose reinforcement for polymer electrolyte membranes with outstanding mechanical properties, Electrochim. Acta, 2011, 57, 104-111.

23 S.-J. Chun, et al., Eco-friendly cellulose nanofiber paperderived separator membranes featuring tunable nanoporous network channels for lithium-ion batteries, $J$. Mater. Chem., 2012, 22(32), 16618-16626.

24 S. Leijonmarck, et al., Single-paper flexible Li-ion battery cells through a paper-making process based on nano- fibrillated cellulose, J. Mater. Chem. A, 2013, 1(15), 46714677.

25 S. J. Eichhorn, et al., Review: current international research into cellulose nanofibres and nanocomposites, J. Mater. Sci., 2010, 45(1), 1-33.

26 T. Saito, et al., Homogeneous Suspensions of Individualized Microfibrils from TEMPO-Catalyzed Oxidation of Native Cellulose, Biomacromolecules, 2006, 7(6), 1687-1691.

27 T. Saito, et al., Individualization of Nano-Sized Plant Cellulose Fibrils by Direct Surface Carboxylation Using TEMPO Catalyst under Neutral Conditions, Biomacromolecules, 2009, 10(7), 1992-1996.

28 A. E. Horvath and T. Lindström, The influence of colloidal interactions on fiber network strength, $J$. Colloid Interface Sci., 2007, 309(2), 511-517.

29 A. Sturcova, G. R. Davies and S. J. Eichhorn, Elastic Modulus and Stress-Transfer Properties of Tunicate Cellulose Whiskers, Biomacromolecules, 2005, 6(2), 1055-1061.

$30 \mathrm{~S}$. Iwamoto, et al., Elastic Modulus of Single Cellulose Microfibrils from Tunicate Measured by Atomic Force Microscopy, Biomacromolecules, 2009, 10(9), 2571-2576.

31 J. J. Peterson, et al., Surface-Grafted conjugated polymers for hybrid cellulose materials, J. Polym. Sci., Part A: Polym. Chem., 2011, 49(14), 3004-3013.

32 S. Katz, R. P. Beatson and A. M. Scallan, The determination of strong and weak acidic groups in sulfite pulps, Sven. Papperstidn., 1984, 87(6), R48-R53.

33 L. Wågberg and R. Hägglund, Kinetics of polyelectrolyte adsorption on cellulosic fibers, Langmuir, 2001, 17(4), 1096-1103.

34 C. C. Sun, Mechanism of moisture induced variations in true density and compaction properties of microcrystalline cellulose, Int. J. Pharm., 2008, 346(1-2), 93-101.

35 A. Carlmark and E. Malmström, Atom Transfer Radical Polymerization from Cellulose Fibers at Ambient Temperature, J. Am. Chem. Soc., 2002, 124(6), 900-901.

$36 \mathrm{M}$. Willgert, et al., Photoinduced free radical polymerization of thermoset lithium battery electrolytes, Eur. Polym. J., 2011, 47(12), 2372-2378.

37 M. Willgert, et al., New structural lithium battery electrolytes using thiol-ene chemistry, Solid State Ionics, 2013, 236, 2229.

38 D. Ståhlberg, et al., Relaxation properties of particle filled coatings: Experimental study and modelling of a screw joint, Prog. Org. Coat., 2006, 55(2), 112-118. 